\title{
Artificial neural network based simplified one day ahead forecasting of solar photovoltaic power generation
}

\author{
Muhammad Asim Munir*, Abraiz Khattak*, Kashif Imran*, Abasin Ulasyar*, Nasim Ullah** \\ Azhar Ul Haq*** and Adam Khan $* * * *$ \\ * Department of Electrical Power Engineering, U.S. Pakistan Centre for Advanced Studies in Energy (USPCASE), National University of \\ Sciences and Technology (NUST), Islamabad, Pakistan \\ ** Department of Electrical Engineering, College of Electrical and Mechanical Engineering (CEME), National University of Sciences and \\ Technology (NUST), Islamabad, Pakistan \\ ** Department of Electrical Engineering, College of Engineering, Taif University KSA, Saudi Arabia \\ *** Department for Management of Science and Technology Development, Ton Duc Thang University, Ho Chi Minh City, Vietnam \\ Faculty of Electrical \& Electronics Engineering, Ton Duc Thang University, Ho Chi Minh City, Vietnam \\ **** Corresponding Author: adamkhan@tdtu.edu.vn
}

$\begin{array}{ll}\text { Submitted } & : 01 / 05 / 2020 \\ \text { Revised } & : 08 / 04 / 2021 \\ \text { Accepted } & : 18 / 05 / 2021\end{array}$

\begin{abstract}
The intermittency of solar energy resources possesses a serious challenge in balancing the power generation and load demand. To enhance the consistency of the system, it is crucial to forecast solar photovoltaic power. Among numerous techniques, Artificial Neural Network (ANN) is an efficient tool that may help simplify this problem. In this study, all 63 combinations of six input parameters, i.e., temperature, dew point, wind speed, cloud cover, relative humidity, and pressure, were applied one by one to ANN to forecast 24 hours ahead PV generation. The power forecast results were obtained based on weather forecast data of 21 days sampled from the recorded forecasted data of 180 days. To quantify the error between predicted and measured solar PV generation, Root Mean Squared Error (RMSE) was used, and the results of different input combinations were compared on basis of this statistical matrix. The analysis showed that the generation is best predicted on two combinations: the first is comprising of temperature, dew point, relative humidity, and cloud cover, while the second consists of all six parameters. And some of the combinations consisting of three parameters also resulted in RMSEs in close proximity of the least error value.
\end{abstract}

Keywords: ANN; Power forecasting; Solar PV; Weather parameters.

\section{INTRODUCTION}

Solar PV is one of the main contributors in the generation of renewable energies as it is potentially cheaper than other renewable sources (IRENA, 2018). However, the variability of solar energy due to varying meteorological conditions affects the reliability of the power system (Ariyaratna et al., 2018). The main challenge in integration of the solar power in electrical grid is balancing the generation and load demand. Thus, in order to handle the uncertainty and fluctuations of output power, it is essential to forecast the solar PV generation (Ahmed and Khalid, 2019). 
In past, many studies were conducted to develop solar PV generation forecast models. For example, an ensemble model was constructed to forecast photovoltaic power for short-term horizons (Zhu et al., 2019). The submodels were trained by K-cross-validation methods. The results showed that the generalization ability of the model was improved with K-cross-validation. The authors (Reikard and Hansen, 2019) tested different methods including regression and frequency models for forecasting solar irradiance, and it was learnt that no model consistently dominated others as the forecast accuracy varied by degree of time variation and the forecast horizons.

The authors ( $\mathrm{Li}$ et al., 2016) presented and analyzed the relative performance of Hidden Markov Model and SVM regression techniques for short-term solar irradiance forecasting. Solar irradiance, air temperature, relative humidity, and wind speed were used as input parameters. The results showed that both the models predicted the solar irradiance with good accuracy for sunny days, but the forecast errors were huge for cloudy days. In (Sharma and Kakkar, 2018), five machine learning based models, namely, FoBA, bagEarthGCV, leapForward, spikeslab, and cubist, were investigated under seasonal effects. The results obtained by performing three types of experiments showed that the quality of data selected for training of the model has significant impact on the forecast accuracy.

Solar radiation forecast accuracy of different types of ANN models was compared in (Wang et al., 2016) based on daily observations of meteorological parameters at 12 stations. The results indicated large differences in model accuracies at different stations. In (Jiang and Dong, 2016), state vector machine was converted into a regularization problem with ridge penalty, and an intelligent optimization model was developed to forecast future solar radiation. The optimal parameters were determined using glowworm swarm optimization. In (Dolara et al., 2015), combination of ANN and clear sky model was used to predict output of a solar power plant with eight months of historical weather and power data. The authors in (Cervone et al., 2017) proposed a methodology by combining ANN and Analog Ensemble models to forecast 72 hours ahead generation of solar power plant located in Italy. They used global solar irradiance, cloud cover, temperature, elevation, solar azimuth, and time as input variables to the forecast model. In comparison, the authors in (Ogliari et al., 2017) investigated the performance of deterministic and hybrid stochastic PV output forecast models with inputs comprising of eight meteorological parameters, while, in (Oneto et al., 2017), data driven techniques like Kernel methods, extreme learning machines, and random forest algorithms were applied to forecast power production for microgrids. In addition to the actual historical power production data, six weather variables were applied to train the models. Artificial neural network was proposed in (Leva et al., 2017) to forecast photovoltaic energy, and its sensitivity was analyzed with respect to input data sets. In (Sangrody et al., 2017), the authors used five input weather variables to assess the forecast accuracy of ANN. They also conducted a sensitivity test to show how the uncertainty in each weather variable forecast affects the solar energy prediction.

Apart from abovementioned individual and ensemble machine leaning algorithms, other statistical methods have also been developed to forecast solar PV output. Statistical principles are incorporated in these methods to establish a relationship between historical power data and future PV power. These methods include wavelet analysis (Eseye et al., 2018, Raza et al., 2017), spectral analysis (Raza et al., 2016), and time series methods (Yang et al., 2018). Although statistical or machine learning algorithms are more complex in implementation, they are preferred over physical methods of forecasting as these methods suffer from lower prediction accuracy while establishing the relationship between solar power and environmental factors (Sobri et al., 2018). A comprehensive review was presented on forecast of solar PV power, and it is observed that the forecast models were mostly based on statistical or machine learning algorithms. Among these forecasting techniques, ANN was the most widely used by the authors (Antonanzas et al., 2016).

The previous research regarding solar power forecasting was focused on the development and performance evaluation of the forecast models based on different machine learning algorithms and statistical techniques. However, ambiguity still remains in the selection of input weather parameters as no standard parameters have been suggested till now. Keeping in view the above motivations, this work is focused on finding the most effective combination of 
weather parameters to be used as input to forecast solar PV generation. This is done by applying all the possible combinations of six input parameters and finding the best one by the analysis of the results.

\section{METHODOLOGY}

The first step in solar PV generation forecasting was acquisition of the forecasted and historical data. In next stage, the errors in the forecasted weather data were calculated to show how the uncertainties in this data would affect the PV output forecast results. The third step was the development of the forecast model.

A typical statistical model to forecast one day ahead solar PV generation is shown in Figure 1. There are two main steps to predict the power output. In the first step, model is trained using historical dataset. This dataset includes observed weather data of the area, where the solar power plant is located and measured generation over a period of time. Usually, the historical data of one year is required to train the model. Once the model is trained, hourly weather forecast of the day is applied to the model for which the generation forecast is required. In this study, artificial neural network based model was implemented with Levenberg-Marquardt backpropagation algorithm. The LM algorithm takes up more memory than other algorithms used with ANN. However, it is preferred over them because of its faster training of the model (Çelik et al., 2016). The model was trained using the observed weather and PV output data of last year. Once the model was trained, forecasted weather was applied as input to the model. This step was repeated for all the combinations of the inputs. In the final step, solar PV generation results were compared on the basis of root mean squared error. All these steps to predict 24 hours ahead PV output are thoroughly explained in the following discussion.

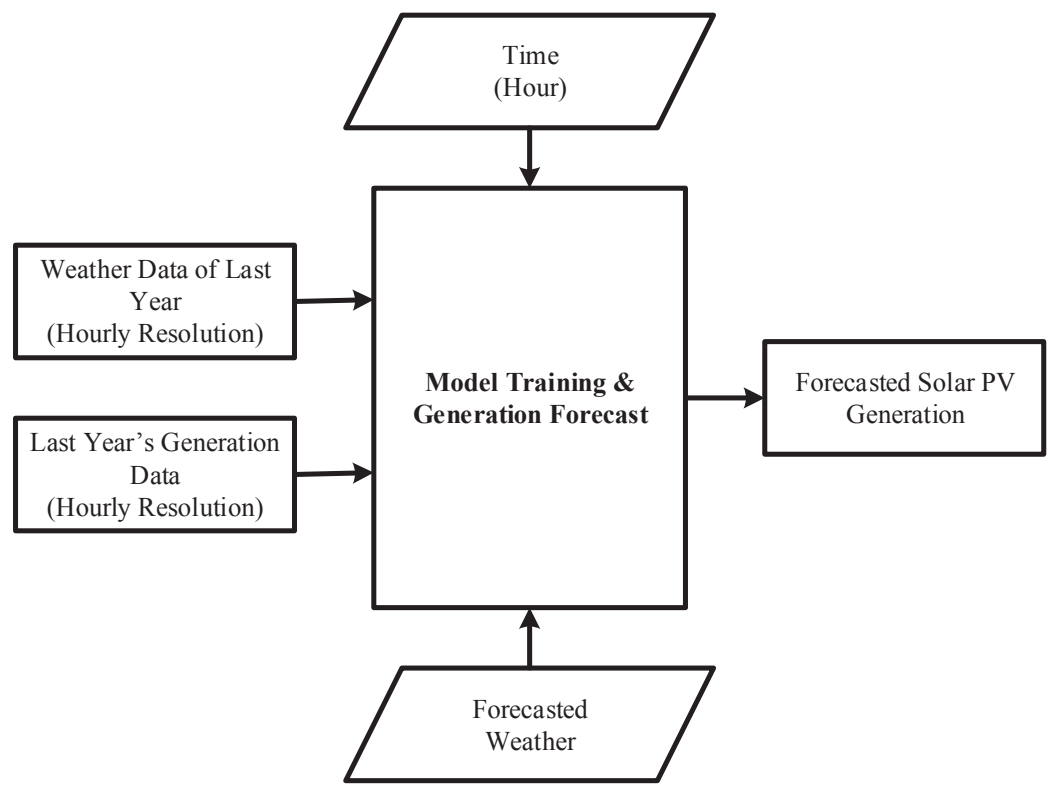

Figure 1. A typical solar PV output forecast model.

\section{Data Acquisition and Analysis}

In this study, power generation data was obtained from a solar power plant located in Lahore for the period from October 17, 2017, to May 14, 2019. The site had 80 solar panels with maximum generation capacity of $20.8 \mathrm{~kW}$. Observed weather data for the abovementioned period was recorded from a weather forecast website 
(WeatherUnderground, 2019). Forecasted weather data for 180 days from October 17, 2018, to May 14, 2019, was recorded from the same website. The website provides weather forecast for next 15 days. But the forecasts were recorded on daily basis as the webpage constantly updates the forecast, and hence, more accurate weather predictions could be recorded. Both the forecasted and historical data were arranged in hourly resolution. Weather parameters provided were temperature, dew point, wind speed, sky cover, pressure, and relative humidity.

To quantify the error in forecasted weather data, it was compared with the observed data of the same 180-day period. Different matrices can be used to calculate the difference between forecasted and observed values, i.e., root mean squared error (RMSE), mean absolute error (MAE), and mean absolute percentage error (Alanazi et al., 2017). In this study, MAE given in Eq. 2 was used to evaluate the error in weather forecasting, and results are shown in Figure 2. It can be seen that the pressure was most accurately forecasted with maximum MAE of 1.77 on day 148, while the least accurate forecast was of sky cover as the calculated MAE exceeds $10 \%$ for 77 days. The MAEs of sky cover range from $0.65 \%$ to $23.21 \%$. The error in forecasted data of other four meteorological parameters lies in the range of 0.61 to 13.48 .
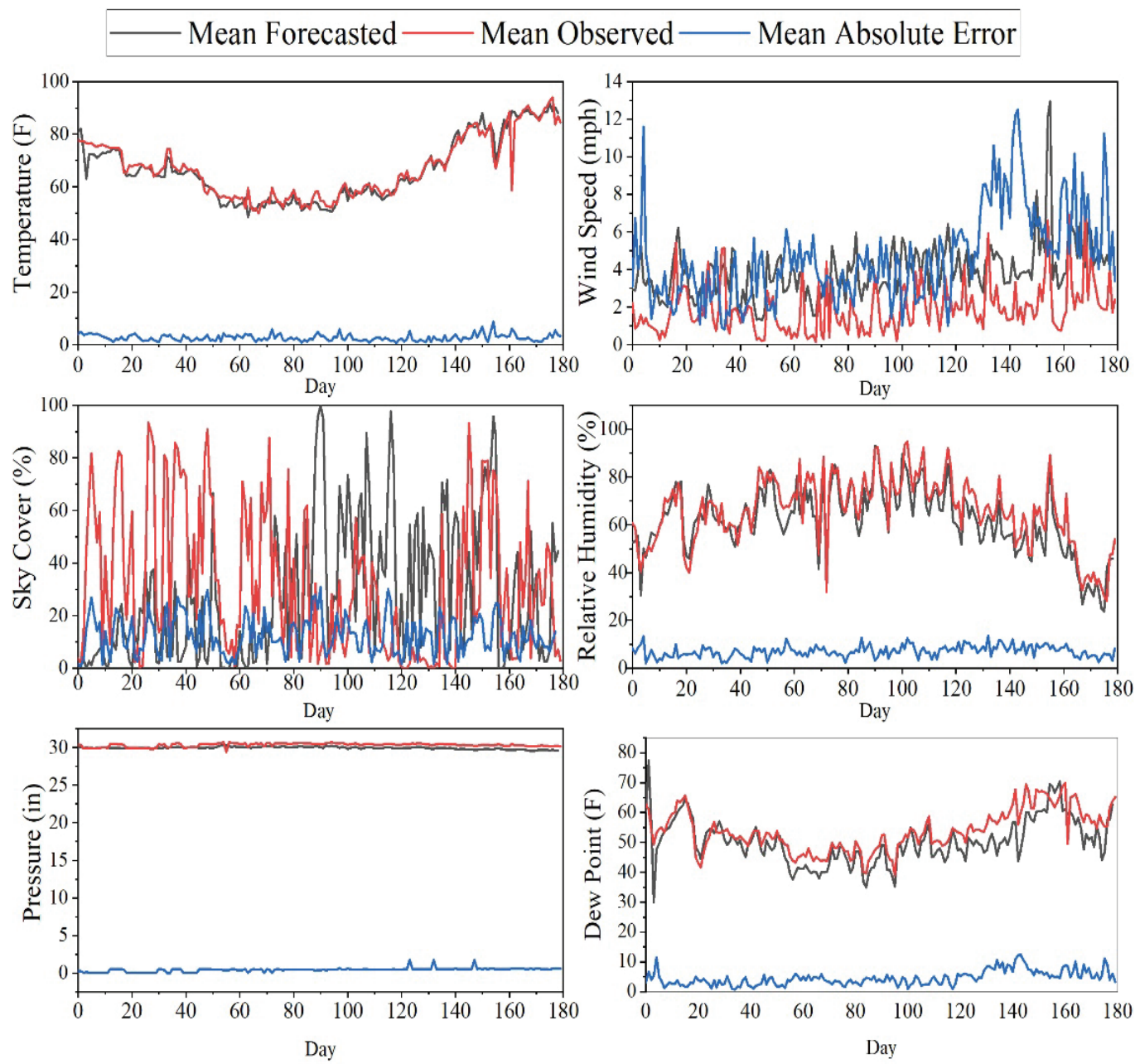

Figure 2. Forecasted and Observed weather data with MAEs. 
In order to simplify the analysis, 21-day set was taken for which the generation would be forecasted. Sampling is done on the basis of the average MAE of all forecasted parameters of a particular day. The data was divided into three groups of 60 days, and seven samples were taken from each group. The samples and the respective errors are shown in Table 1. Among each set of 7 samples, the first two are the days with the lowest error in that particular group, and the last two are with the highest error, and in between them are those three samples that are taken randomly. The sampling criteria were carefully designed, so that the samples with a wider range of forecast errors could be included in the study. The actual PV generation of these 21 sampled days is shown in Figure 3.

Table 1. Samples from forecasted data of 180 days.

\begin{tabular}{|c|c|c|c|c|c|c|c|c|}
\hline \multicolumn{3}{|c|}{17 Oct 2018 -20 Dec 2018} & \multicolumn{3}{|c|}{21 Dec 2018 - 06 Mar 2019} & \multicolumn{3}{|c|}{07 Mar 2019 - 14 May 2019} \\
\hline \multicolumn{3}{|c|}{ Data Set 1 (d1 - d60) } & \multicolumn{3}{|c|}{ Data Set $2(d 61-d 120)$} & \multicolumn{3}{|c|}{ Data Set $3(d 121$ - d180) } \\
\hline Day & Date & Error & Day & Date & Error & Day & Date & Error \\
\hline 1 & $19-11-18$ & 1.35 & 8 & $10-01-19$ & 1.69 & 15 & 05-05-19 & 2.04 \\
\hline 2 & $28-11-18$ & 1.36 & 9 & $19-02-19$ & 1.82 & 16 & 03-05-19 & 2.50 \\
\hline 3 & $25-10-18$ & 2.18 & 10 & $21-12-18$ & 3.26 & 17 & $15-03-19$ & 3.22 \\
\hline 4 & $09-11-18$ & 2.30 & 11 & 07-01-19 & 2.00 & 18 & 03-04-19 & 3.82 \\
\hline 5 & $11-12-18$ & 1.90 & 12 & 02-03-19 & 2.91 & 19 & 24-04-19 & 3.69 \\
\hline 6 & $17-12-18$ & 4.04 & 13 & 19-01-19 & 3.71 & 20 & $20-03-19$ & 5.13 \\
\hline 7 & $21-10-18$ & 5.23 & 14 & 03-02-19 & 3.85 & 21 & 16-04-19 & 5.22 \\
\hline
\end{tabular}

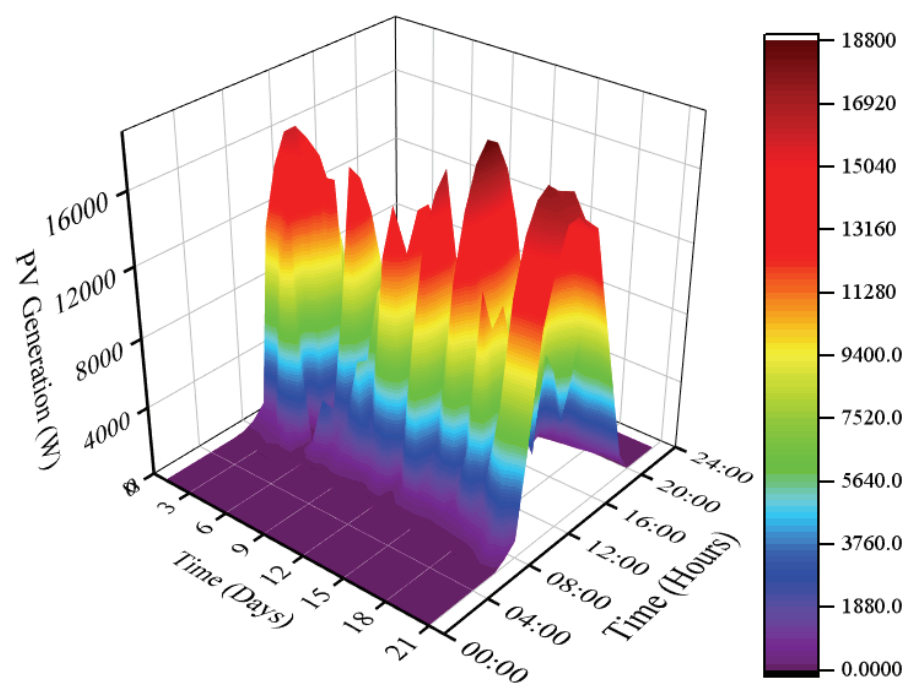

Figure 3. Actual PV generation of 21 sampled days. 


\section{Performance Matrices}

Difference between measured and predicted PV output on a particular day was calculated by root mean squared error. The forecast results for all input combinations were compared on the basis of RMSE given by

$R M S E=\sqrt{\frac{1}{N} \sum_{i=1}^{N}\left(y_{i}-\hat{y}_{i}\right)^{2}}$

Weather forecast error was quantified by mean absolute error for all weather parameters.

$M A E=\frac{\sum_{i=1}^{N}\left|y_{i}-\hat{y}_{i}\right|}{N}$

To analyze the statistical relationship between weather parameters and solar PV generation, correlation coefficient is used. This degree of correspondence between two variables is given by the correlation coefficient that lies in the range of -1 to 1 .

$r=\frac{\sum(\mathrm{y} \cdot \hat{y})-\left(\sum y\right)\left(\sum \hat{y}\right)}{\sqrt{\left[N \sum y^{2}-\left(\sum y\right)^{2}\right]\left[N \sum \hat{y}^{2}-\left(\sum \hat{y}\right)^{2}\right]}}$

In the above equations, $\mathrm{y}$ represents the observed value, and $\hat{y}$ is the predicted value at a particular hour. $\mathrm{N}$ represents the total hours of a day.

\section{Correlation Among Solar PV Generation and Weather Parameters}

Correlation analysis among solar PV generation and six weather parameters is shown in Figure 4. The higher the value of correlation coefficient between two variables, the stronger the correlation between them. It is evident from the correlation coefficients values that the dew point, wind speed, and pressure are not strongly correlated with solar PV generation. These parameters have coefficients of $0.18,-0.09$, and -0.05 , respectively. On the other hand, solar PV output has a correlation of 0.42 with temperature and high negative correlation of -0.61 and -0.58 with relative humidity and sky cover, respectively. The negative values indicate that PV generation decreases with the increase in these weather parameters. But that would not always be true because the other factors would also contribute to the generation. Therefore, input parameters to the forecast model cannot be solely selected on the basis of correlation coefficients (Qing and Niu, 2018). The analysis of correlation coefficients shows that the relative humidity has higher correlation with solar PV generation than other meteorological parameters. This corresponds to the findings of sensitivity test conducted by (Sangrodi et al., 2017). They concluded from the test that the forecast accuracy of the ANN based model could be enhanced with accurate forecast data of relative humidity as this parameter highly affected the generation. 

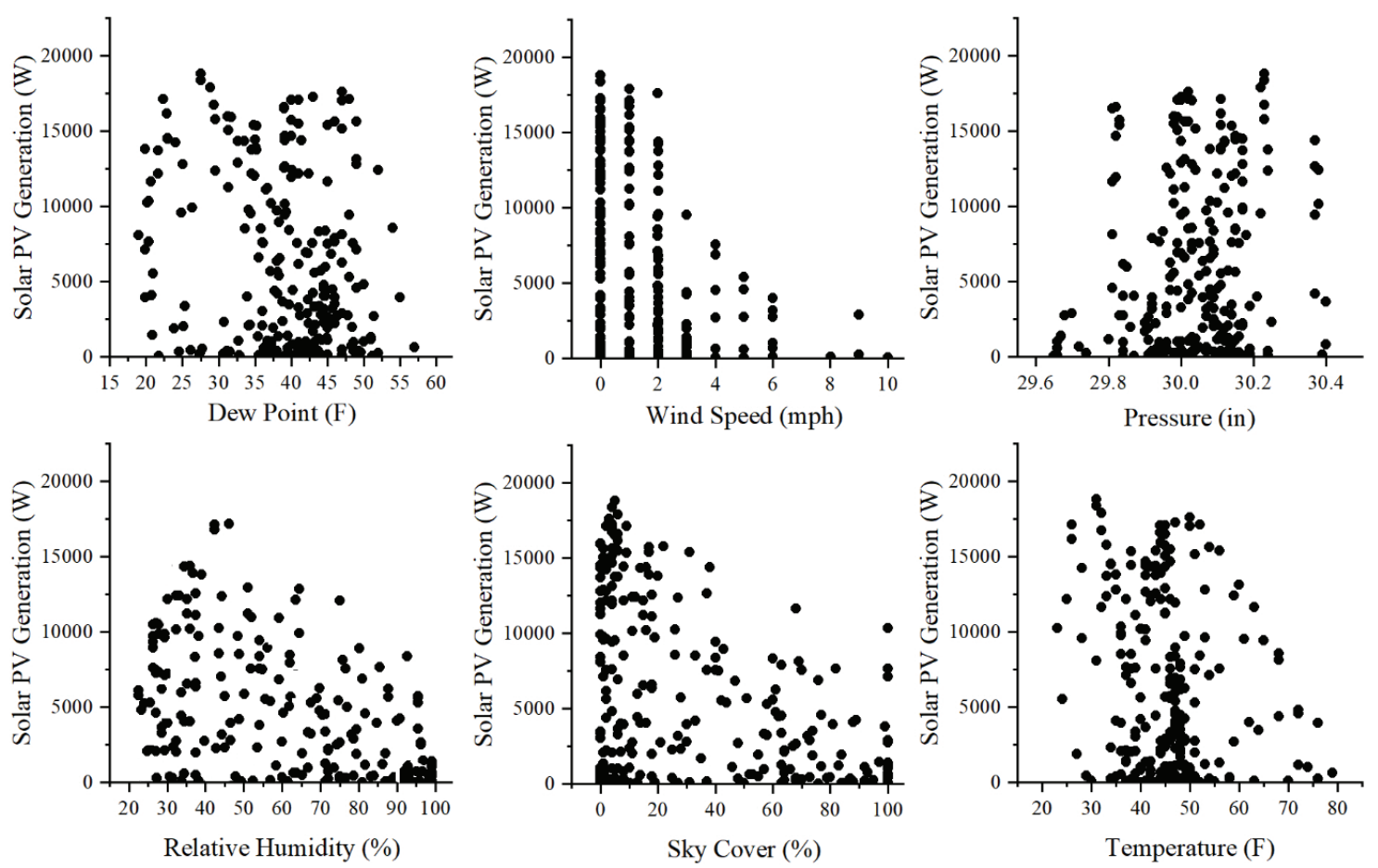

Figure 4. Correlation between weather parameters and PV generation.

\section{Application of Artificial Neural Network}

There are three stages for implementing a neural network. In the first stage, the numbers of hidden layers, neurons, input, and output parameters are defined. A hidden layer feeds the data to the next layer through a set of weights that are initialized randomly at start. The second stage is the training of the model based on the training parameters, i.e., no. of iterations, validation checks, and learning rate. The third and final stage is the validation phase, where the inputs against which the outputs are known, excluding those used in the training phase, are applied to test the accuracy of the model. If the accuracy corresponds to the set goal, further training is not required; otherwise, the training continues (Wang et al., 2019). One of the things that differentiates ANN from other machine learning approaches is its learning ability. The model is initialized with random weights. A training data set is applied to ANN that consists of the inputs and the actual outputs against those inputs. The algorithms find the best fit between input and output by updating weights on neurons in each iteration (Yegnanarayana, 2009). Weight is updated by

$w_{i j}^{\text {new }}=w_{j i}^{\text {previous }}-\eta \frac{\sigma E}{w_{j i}}$

where $w$ is weight between $\mathrm{i}^{\text {th }}$ and $\mathrm{j}^{\text {th }}$ neuron, and $\eta$ is the learning rate. $\mathrm{E}$ is the error calculated by

$$
E=\sum_{k=1}^{L} \sum_{j=1}^{q}\left(b_{k j}-z_{k j}\right)^{2}
$$


where $b_{k j}$ is the observed value, and $z_{k j}$ is the predicted value. In this paper, ANN methodology was simulated in Matlab software using Levenberg-Marquardt backpropagation algorithm, and optimal weights have been evaluated. Additionally, logarithmic-sigmoid functions were used during training procedure. Two numbers of layers with different neurons were implemented to train the forecast model. The simulation process is illustrated in Figure 5.

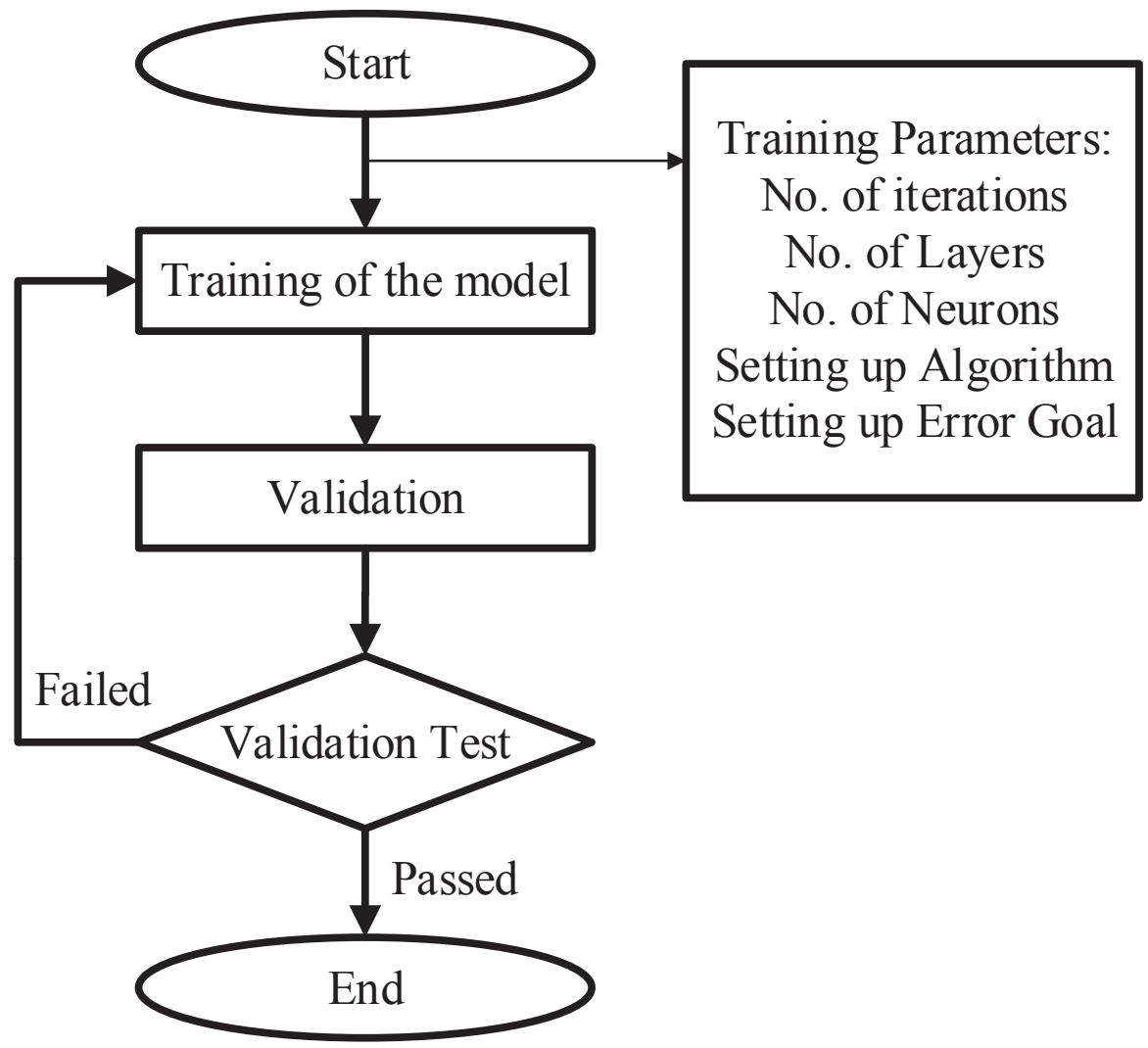

Figure 5. Simulation process of ANN.

\section{Finding the Optimal Number of Neurons}

Before applying ANN for all the combinations, the number of neurons was carefully chosen for each category of the combinations as the neurons in the hidden layers have significant impact on forecast results of ANN. There is no specific rule to determine the optimal no. of neurons for ANN, and the selection is solely based on trial and error. In this study, the forecast model was simulated for different number of input parameters; therefore, the number of neurons would vary accordingly. Simulations were performed on one combination from each of the six input groups. For a particular input combination, the model was trained with different number of neurons ranging from 5 to 50 in either of the layer and solar PV generation was forecasted in each case. The same number of neurons was incorporated in both layers. The results were compared on the basis of RMSE between the forecasted and actual PV generation. Figure 6 plots the RMSE versus the number of neurons. The optimal number of neurons for individual and combination of 2 to 6 parameters came out to be 35,20,25, 50,50, and 45, respectively. 

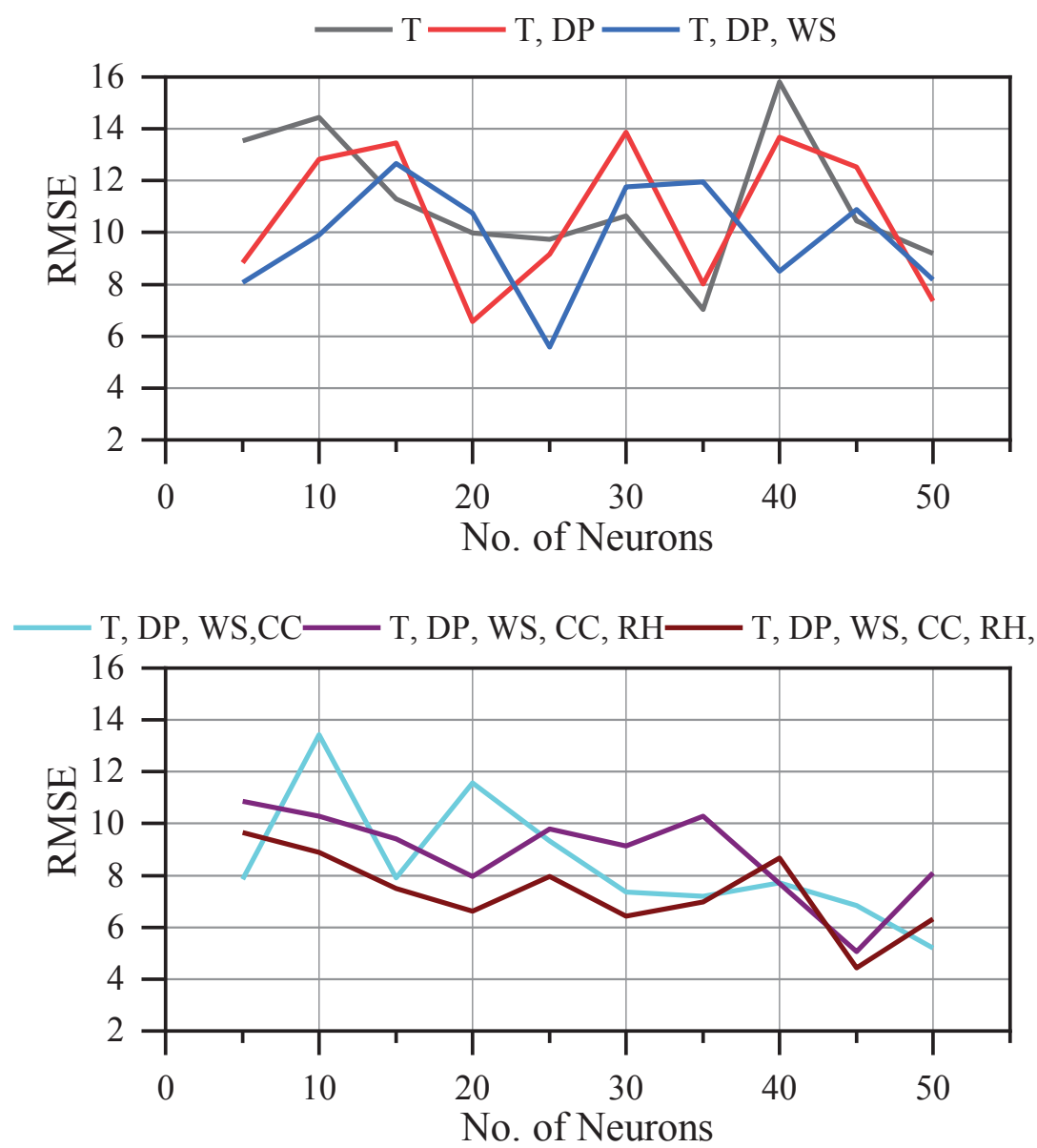

Figure 6. Variation in RMSE w.r.t number of neurons for each category of combinations.

\section{RESULTS AND DISCUSSION}

After selection of optimal number of neurons, a series of simulations were performed by first applying the six input parameters, i.e., temperature, dew point, relative humidity, wind speed, cloud cover, and pressure individually to the forecast model and then in the combinations of 2, 3, and 6 variables. Simulation results were obtained for 21 days sampled from 180 days, for which the forecasted weather data was recorded, and RMSEs are presented in Figure 7. Historical weather and power data of last year were applied for training the model, and power generation is forecasted day by day for all the 63 possible combinations. The results were analyzed on the basis of Root Mean Square Error.

Firstly, the impact of varying the number of variables was assessed. The results shown in Table 2 are divided into six categories with respect to the number of input variables applied. When individual parameters were taken as input, cloud cover came out to be the best predictor of the PV generation with an RMSE of 6.91. The least impact in this category was of pressure that showed an RMSE of 13.09. With combinations of two weather parameters as input, combination of temperature and cloud cover gave the lowest RMSE of 5.53 and reduced the root mean squared error by $20 \%$ from the best results of the previous category. 
The generation forecast errors were further reduced when input parameters were increased to three. Among all the combinations of three variables, the combination with temperature, cloud cover, and relative humidity had the minimum RMSE of 4.49 , which was an improvement of $18.8 \%$. Similarly, the combinations of four variables were applied to the model as input, and the results showed that the group of temperature, dew point, cloud cover, and relative humidity had the lowest RMSE of 4.44 . There was a slight reduction of $1.11 \%$ from the minimum error of the previous results.

Increasing the number of input parameters from four to five changed the trend as the least root mean squared errors of this category were increased from the previous one. Temperature, wind speed, cloud cover, relative humidity, and pressure were the best predictors in this category with an error of 4.48. Application of all six variables to the forecast model predicted the solar PV generation with an RMSE of 4.44.

Another aspect of analysis of the results is to simplify the PV generation forecast model in terms of input parameters. For this, all those combinations are discussed, which resulted in errors in the range of 4.44 to 4.6. The highest accuracy of the forecast results was found with two sets of combinations. The use of all six parameters and the combination of temperature, dew point, cloud cover, and relative humidity gave the least RMSE of 4.44 among the 63 input combinations. Combinations 60 and 61 are the only five-parameter combinations that resulted in RMSE falling in the above defined range. There are 6 sets of four inputs, whose forecast accuracies were in proximity of the best predicted results. These combinations are 45, 46, 48, 49, 51, and 52 with RMSEs in the range of 4.44 to 4.54. Among the combinations of three input parameters, it can be seen that six sets, i.e., 26, 29, 30, 36, 38, and 39 have RMSE that fall in the aforementioned range.

Although the six parameters and one of the four variables combination best predict the PV output, the analysis implies that any of the combinations mentioned above with RMSE between 4.44 and 4.6 can be used to forecast the generation. In order to simplify the model, the three-variable combinations can be used instead of four, five, or six inputs. The analysis of the models proposed in literature also shows that the simplified model can produce better results in some cases. For example, Ogliari et al., (2017) analyzed the performance of forecast models with inputs comprising of three and five parameters. The forecast errors indicated that the three-input-parameter model outperformed the other one. In another study, PV forecast model based on k-nearest neighbor neural network with five input parameters was proposed by Zhang et al. (2015), and it resulted in higher RMSE of 8.72. Similarly, Graditi et al. (2016) developed an ANN based prediction model having four meteorological variables as inputs. The average RMSE obtained by the model was 6.66 .

Table 2. Overall RMSEs of 21 days.

\begin{tabular}{|c|c|c|c|c|c|c|c|c|}
\hline No. & Combination & RMSE & No. & Combination & RMSE & No. & Combination & RMSE \\
\hline 1 & $\mathrm{~T}$ & 7.04 & 22 & $\mathrm{~T}, \mathrm{DP}, \mathrm{WS}$ & 5.57 & 42 & $\mathrm{~T}, \mathrm{DP}, \mathrm{WS}, \mathrm{CC}$ & 5.20 \\
\hline 2 & $\mathrm{DP}$ & 8.73 & 23 & $\mathrm{~T}, \mathrm{DP}, \mathrm{CC}$ & 4.62 & 43 & $\mathrm{~T}, \mathrm{DP}, \mathrm{WS}, \mathrm{RH}$ & 5.09 \\
\hline 3 & $\mathrm{WS}$ & 8.20 & 24 & $\mathrm{~T}, \mathrm{DP}, \mathrm{RH}$ & 6.61 & 44 & $\mathrm{~T}, \mathrm{DP}, \mathrm{WS}, \mathrm{P}$ & 5.16 \\
\hline 4 & $\mathrm{CC}$ & 6.91 & 25 & $\mathrm{~T}, \mathrm{DP}, \mathrm{P}$ & 5.89 & 45 & $\mathrm{~T}, \mathrm{DP}, \mathrm{CC}, \mathrm{RH}$ & 4.44 \\
\hline
\end{tabular}




\begin{tabular}{|c|c|c|c|c|c|c|c|c|}
\hline 5 & RH & 7.86 & 26 & $\mathrm{~T}, \mathrm{WS}, \mathrm{CC}$ & 4.59 & 46 & $\mathrm{~T}, \mathrm{DP}, \mathrm{CC}, \mathrm{P}$ & 4.54 \\
\hline 6 & $\mathrm{P}$ & 13.09 & 27 & T, WS, RH & 5.09 & 47 & $\mathrm{~T}, \mathrm{DP}, \mathrm{RH}, \mathrm{P}$ & 5.38 \\
\hline 7 & $\mathrm{~T}, \mathrm{DP}$ & 6.56 & 28 & $\mathrm{~T}, \mathrm{WS}, \mathrm{P}$ & 5.55 & 48 & $\mathrm{~T}, \mathrm{WS}, \mathrm{CC}, \mathrm{RH}$ & 4.49 \\
\hline 8 & $\mathrm{~T}, \mathrm{WS}$ & 6.78 & 29 & $\mathrm{~T}, \mathrm{CC}, \mathrm{RH}$ & 4.49 & 49 & $\mathrm{~T}, \mathrm{WS}, \mathrm{CC}, \mathrm{P}$ & 4.51 \\
\hline 9 & $\mathrm{~T}, \mathrm{CC}$ & 5.53 & 30 & $\mathrm{~T}, \mathrm{CC}, \mathrm{P}$ & 4.51 & 50 & T, WS, RH, P & 5.75 \\
\hline 10 & $\mathrm{~T}, \mathrm{RH}$ & 6.97 & 31 & $\mathrm{~T}, \mathrm{RH}, \mathrm{P}$ & 5.33 & 51 & $\mathrm{~T}, \mathrm{CC}, \mathrm{RH}, \mathrm{P}$ & 4.49 \\
\hline 11 & $\mathrm{~T}, \mathrm{P}$ & 7.00 & 32 & $\mathrm{DP}, \mathrm{WS}, \mathrm{CC}$ & 4.71 & 52 & $\mathrm{DP}, \mathrm{WS}, \mathrm{CC}, \mathrm{RH}$ & 4.46 \\
\hline 12 & DP, WS & 7.05 & 33 & DP, WS, RH & 5.47 & 53 & DP, WS, CC, P & 4.62 \\
\hline 13 & $\mathrm{DP}, \mathrm{CC}$ & 6.21 & 34 & DP, WS, P & 5.81 & 54 & DP, WS, RH, P & 5.89 \\
\hline 14 & $\mathrm{DP}, \mathrm{RH}$ & 6.70 & 35 & $\mathrm{DP}, \mathrm{CC}, \mathrm{RH}$ & 4.62 & 55 & $\mathrm{DP}, \mathrm{CC}, \mathrm{RH}, \mathrm{P}$ & 4.92 \\
\hline 15 & DP, $P$ & 7.24 & 36 & $\mathrm{DP}, \mathrm{CC}, \mathrm{P}$ & 4.51 & 56 & WS, CC, RH, P & 4.74 \\
\hline 16 & WS, CC & 5.85 & 37 & $\mathrm{DP}, \mathrm{RH}, \mathrm{P}$ & 5.47 & 57 & T, DP, WS, CC, RH & 5.07 \\
\hline 17 & WS, RH & 6.48 & 38 & WS, CC, RH & 4.51 & 58 & T, DP, WS, CC, P & 4.62 \\
\hline 18 & WS, P & 6.92 & 39 & WS, CC, P & 4.55 & 59 & T, DP, WS, RH, P & 5.33 \\
\hline 19 & $\mathrm{CC}, \mathrm{RH}$ & 5.56 & 40 & WS, RH, P & 5.23 & 60 & $\mathrm{~T}, \mathrm{DP}, \mathrm{CC}, \mathrm{RH}, \mathrm{P}$ & 4.57 \\
\hline 20 & $\mathrm{CC}, \mathrm{P}$ & 5.56 & 41 & $\mathrm{CC}, \mathrm{RH}, \mathrm{P}$ & 4.83 & 61 & T, WS, CC, RH, P & 4.48 \\
\hline \multirow[t]{2}{*}{21} & RH, P & 6.77 & & & & 62 & DP, WS, CC, RH, P & 4.64 \\
\hline & & & & & & 63 & $\begin{array}{c}\mathrm{T}, \mathrm{DP}, \mathrm{WS}, \mathrm{CC}, \mathrm{RH}, \\
\mathrm{P}\end{array}$ & 4.44 \\
\hline
\end{tabular}



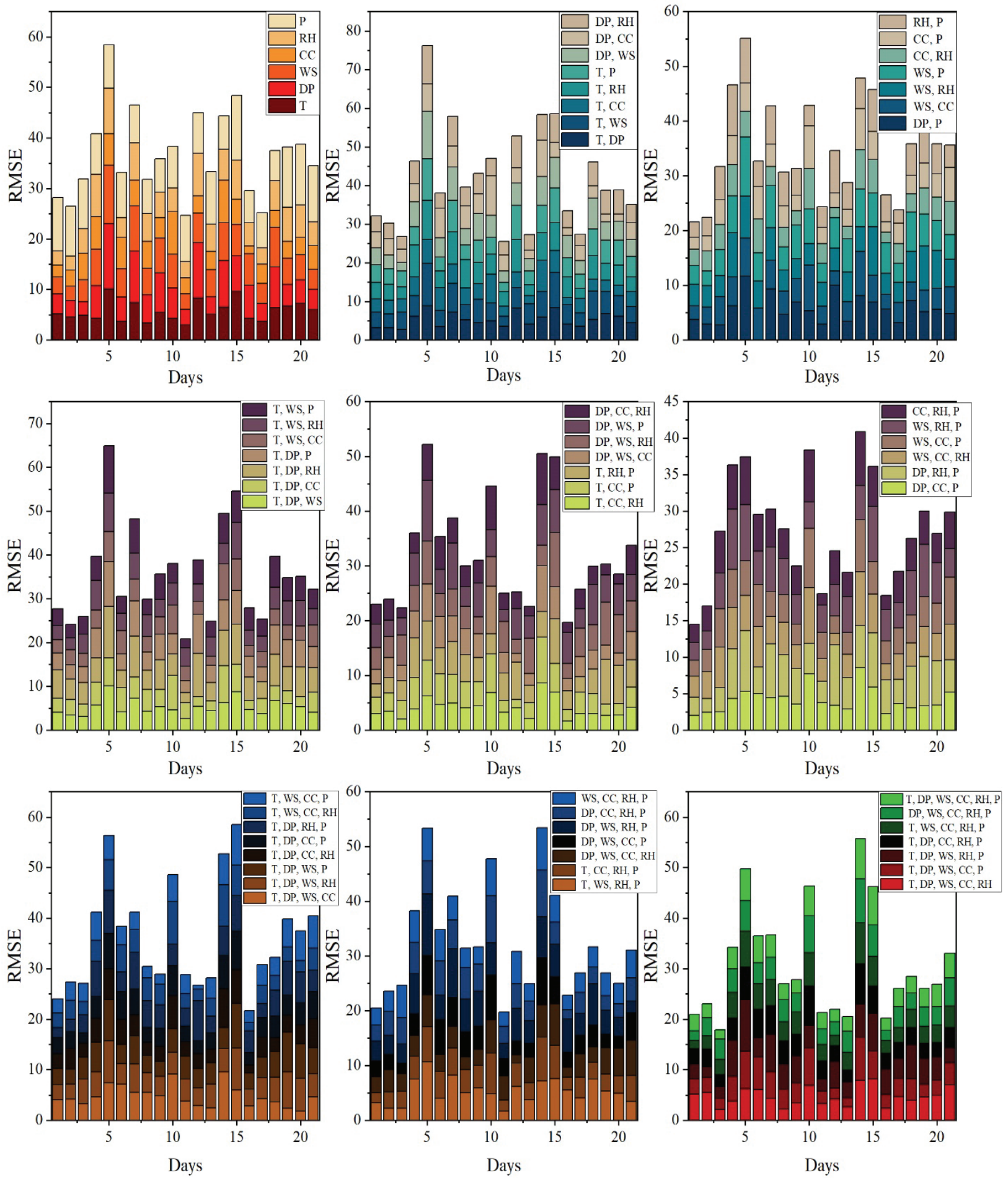

Figure 7. RMSEs of forecasted generation for all combinations. 


\section{CONCLUSION}

The application of 63 input combinations of six weather parameters to the solar PV generation forecast model was investigated. The model was based on Artificial Neural Network with Levenberg-Marquardt Backpropagation algorithm and trained using one year of historical weather and power generation data. The power was forecasted for 21 days for each input combination, and the results were compared on the basis of root mean squared error. Applying the individual parameters one by one as input, the lowest RMSE came out to be $6.91 \%$ with cloud cover as input. Then, the combinations of more than one parameter were applied to the model. Among the sets of two parameters, the combination of temperature and cloud cover gave an RMSE of $5.53 \%$. The most accurate predictor in the set of four parameters combinations were the group of temperature, dew point, cloud cover, and relative humidity. RMSE for this combination was $4.44 \%$, which was the least RMSE value not only in this set, but also among all the combinations. Among three-input combinations, the one comprising of temperature, cloud cover, and relative humidity predicted the solar PV generation with accuracy in proximity to the best obtained results mentioned above. It is concluded based on the 180 days forecasted and measured data fed to the ANN with Levenberg-Marquardt Backpropagation algorithm trained on one year of historical data; the four parameters (temperature, dew point, cloud cover, and relative humidity) were found to be sufficient for one day ahead forecasting of solar PV output power.

\section{FUNDING}

This research was supported by Taif University Researchers Supporting Project number (TURSP-2020/144), Taif University, Taif, Saudi Arabia.

\section{ACKNOWLEDGMENT}

The authors acknowledge the funding of Researchers Supporting Project number (TURSP-2020/144), Taif University, Taif, Saudi Arabia.

\section{REFERENCES}

Ahmed, A., and M. Khalid. 2019. A review on the selected applications of forecasting models in renewable power systems. Renewable and Sustainable Energy Reviews 100: 9-21.

Alanazi, M., M. Mahoor, and A. Khodaei. Year. Published. Two-stage hybrid day-ahead solar forecasting, pp. 1-6. In, 2017 North American Power Symposium (NAPS), 2017. IEEE.

Antonanzas, J., N. Osorio, R. Escobar, R. Urraca, F. J. Martinez-de-Pison, and F. Antonanzas-Torres. 2016. Review of photovoltaic power forecasting. Solar Energy 136: 78-111.

Ariyaratna, P., K. M. Muttaqi, and D. Sutanto. 2018. A novel control strategy to mitigate slow and fast fluctuations of the voltage profile at common coupling Point of rooftop solar PV unit with an integrated hybrid energy storage system. Journal of Energy Storage 20: 409-417.

Çelik, Ö., A. Teke, and H. B. Yıldırım. 2016. The optimized artificial neural network model with LevenbergMarquardt algorithm for global solar radiation estimation in Eastern Mediterranean Region of Turkey. Journal of cleaner production 116: 1-12.

Cervone, G., L. Clemente-Harding, S. Alessandrini, and L. Delle Monache. 2017. Short-term photovoltaic power forecasting using Artificial Neural Networks and an Analog Ensemble. Renewable Energy 108: 274286. 
Dolara, A., F. Grimaccia, S. Leva, M. Mussetta, and E. Ogliari. 2015. A physical hybrid artificial neural network for short term forecasting of PV plant power output. Energies 8: 1138-1153.

Eseye, A. T., J. Zhang, and D. Zheng. 2018. Short-term photovoltaic solar power forecasting using a hybrid Wavelet-PSO-SVM model based on SCADA and Meteorological information. Renewable energy 118: $357-$ 367.

Graditi, G., S. Ferlito, and G. Adinolfi. 2016. Comparison of Photovoltaic plant power production prediction methods using a large measured dataset. Renewable energy 90: 513-519.

IRENA. 2018. Renewable Energy Statistics, Abu Dhabi, UAE,.

Jiang, H., and Y. Dong. 2016. A nonlinear support vector machine model with hard penalty function based on glowworm swarm optimization for forecasting daily global solar radiation. Energy conversion and management 126: 991-1002.

Leva, S., A. Dolara, F. Grimaccia, M. Mussetta, and E. Ogliari. 2017. Analysis and validation of 24 hours ahead neural network forecasting of photovoltaic output power. Mathematicsand computers in simulation 131: 88-100.

Li, J., J. K. Ward, J. Tong, L. Collins, and G. Platt. 2016. Machine learning for solar irradiance forecasting of photovoltaic system. Renewable energy 90: 542-553.

Ogliari, E., A. Dolara, G. Manzolini, and S. Leva. 2017. Physical and hybrid methods comparison for the day ahead PV output power forecast. Renewable energy 113: 11-21.

Oneto, L., F. Laureri, M. Robba, F. Delfino, and D. Anguita. 2017. Data-driven photovoltaic power production nowcasting and forecasting for polygeneration microgrids. IEEE Systems Journal 12: 2842-2853.

Qing, X., and Y. Niu. 2018. Hourly day-ahead solar irradiance prediction using weather forecasts by LSTM. Energy 148: 461-468.

Raza, M. Q., M. Nadarajah, and C. Ekanayake. 2017. Demand forecast of PV integrated bioclimatic buildings using ensemble framework. Applied energy 208: 1626-1638.

Raza, M. Q., M. Nadarajah, and C. Ekanayake. 2016. On recent advances in PV output power forecast. Solar Energy 136: 125-144.

Reikard, G., and C. Hansen. 2019. Forecasting solar irradiance at short horizons: Frequency and time domain models. Renewable energy 135: 1270-1290.

Sangrody, H., M. Sarailoo, N. Zhou, N. Tran, M. Motalleb, and E. Foruzan. 2017. Weather forecasting error in solar energy forecasting. IET Renewable Power Generation 11: 1274-1280.

Sharma, A., and A. Kakkar. 2018. Forecasting daily global solar irradiance generation using machine learning. Renewable and Sustainable Energy Reviews 82: 2254-2269.

Sobri, S., S. Koohi-Kamali, and N. A. Rahim. 2018. Solar photovoltaic generation forecasting methods: A review. Energy Conversion and Management 156: 459-497.

Wang, F., Z. Zhang, C. Liu, Y. Yu, S. Pang, N. Duić, M. Shafie-Khah, and J. P. Catalao. 2019. Generative adversarial networks and convolutional neural networks based weather classification model for day ahead short-term photovoltaic power forecasting. Energy conversion and management 181: 443-462.

Wang, L., O. Kisi, M. Zounemat-Kermani, G. A. Salazar, Z. Zhu, and W. Gong. 2016. Solar radiation prediction using different techniques: model evaluation and comparison. Renewable and Sustainable Energy Reviews 61: 384-397.

WeatherUnderground. 2019. Weather Forecast \& Reports - Long Range \& Local. Available Online: https://www.wunderground.com/. [Accessed: 27-Aug-2019]. 
Yang, D., J. Kleissl, C. A. Gueymard, H. T. Pedro, and C. F. Coimbra. 2018. History and trends in solar irradiance and PV power forecasting: A preliminary assessment and review using text mining. Solar Energy 168: 60-101.

Yegnanarayana, B. 2009. Artificial neural networks, PHI Learning Pvt. Ltd.

Zhang, Y., M. Beaudin, R. Taheri, H. Zareipour, and D. Wood. 2015. Day-ahead power output forecasting for small-scale solar photovoltaic electricity generators. IEEE Transactions on Smart Grid 6: 2253-2262.

Zhu, R., W. Guo, and X. Gong. 2019. Short-Term Photovoltaic Power Output Prediction Based on k-Fold CrossValidation and an Ensemble Model. Energies 12: 1220. 